\title{
FROM HERMITE POLYNOMIALS TO MULTIFRACTIONAL PROCESSES
}

\author{
RENAUD MARTY,* Université de Lorraine
}

\begin{abstract}
We consider a class of multifractional processes related to Hermite polynomials. We show that these processes satisfy an invariance principle. To prove the main result of this paper, we use properties of the Hermite polynomials and the multiple Wiener integrals. Because of the multifractionality, we also need to deal with variations of the Hurst index by means of some uniform estimates.
\end{abstract}

Keywords: Multifractional process; Hermite polynomial; limit theorem; sample path properties

2010 Mathematics Subject Classification: Primary 60F17; 60G17; 60G22

\section{Introduction}

Hermite processes have attracted a lot of attention for many years because they have nice properties and generalize fractional Brownian motion [7], [14], [17]. Let $m \in \mathbb{N}^{*}$ and $H \in\left(\frac{1}{2}, 1\right)$. The Hermite process $W_{m, H}$ of order $m$ and Hurst index $H$ can be defined in terms of Wiener-Itô-Dobrushin integrals [6] by

$$
W_{m, H}: t \mapsto W_{m, H}(t)=\int_{\mathbb{R}^{m}} f_{m, H}\left(x_{1}, \ldots, x_{m}, t\right) \mathrm{d} \hat{B}_{x_{1}} \cdots \mathrm{d} \hat{B}_{x_{m}}
$$

with the function $f_{m, H}$ given for every $t$ in $\mathbb{R}$ and almost every $\left(x_{1}, \ldots, x_{m}\right)$ in $\mathbb{R}^{m}$ by

$$
f_{m, H}\left(x_{1}, \ldots, x_{m}, t\right)=C(m, H) \frac{\exp \left(\mathrm{i} t\left(x_{1}+\cdots+x_{m}\right)\right)-1}{\mathrm{i}\left(x_{1}+\cdots+x_{m}\right)\left|x_{1} \cdots x_{m}\right|^{(2 H-2+m) / 2 m}},
$$

where $C(m, H)$ is a normalizing constant and $\mathrm{d} \hat{B}$ is a complex Gaussian measure such that (1.1) defines a real process. Note that, for $m=1$, the Hermite process $W_{1, H}$ is the fractional Brownian motion with Hurst index $H$. Moreover, for every integer $m \geq 2$, the Hermite process $W_{m, H}$ and fractional Brownian motion share many properties. For instance, $W_{m, H}$ is selfsimilar with parameter $H$ : for every $a>0$, the process $\left\{W_{m, H}(a t)\right\}_{t \geq 0}$ is equal in distribution to $\left\{a^{H} W_{m, H}(t)\right\}_{t \geq 0}$. It is also a basic model for long-range dependence: the sequence of its increments $\delta W_{m, H}=\left\{\delta W_{m, H}(j)=W_{m, H}(j+1)-W_{m, H}(j)\right\}_{j \in \mathbb{N}}$ is stationary and satisfies the long-range property

$$
\mathbb{E}\left[\delta W_{m, H}(0) \delta W_{m, H}(j)\right] \sim \frac{c_{m, H}}{j^{2-2 H}} \quad \text { as } j \rightarrow \infty,
$$

where $c_{m, H}$ is a positive constant.

Received 14 June 2011; revision received 16 October 2012.

* Postal address: Institut Élie Cartan, Université de Lorraine, B.P. 70239, F-54506 Vandoeuvre-lès-Nancy Cedex, France. Email address: renaud.marty@univ-lorraine.fr 
The invariance principle [5], [7], [16], [17] is one of the most important properties of Hermite processes for applications. It can be stated as follows. Let $X=\left\{X_{j}\right\}_{j \in \mathbb{N}}$ be a Gaussian stationary sequence of centered random variables with variance 1 . We assume that there exists a positive constant $c$ such that $X$ satisfies the long-range property

$$
\mathbb{E}\left[X_{0} X_{j}\right] \sim \frac{c}{j^{2(1-H) / m}} \quad \text { as } j \rightarrow \infty .
$$

We consider a real-valued function $\phi$ satisfying $\int_{\mathbb{R}} \phi(x)^{2} \mathrm{e}^{-x^{2} / 2} \mathrm{~d} x<\infty$ and with Hermite rank equal to $m$. Roughly speaking, this means that there exists a sequence of real numbers $\left\{\phi_{j}\right\}_{j \geq m}$ such that $\phi_{m} \neq 0$ and $\phi=\sum_{j=m}^{\infty} \phi_{j} P_{j}$, where, for each $j \geq m, P_{j}$ is the $j$ th Hermite polynomial. We define the sequence of processes $\left\{S_{\phi, H}^{N}\right\}_{N \in \mathbb{N}}$ by

$$
S_{\phi, H}^{N}(t)=\frac{1}{N^{H}} \sum_{j=1}^{\lfloor N t\rfloor} \phi\left(X_{j}\right)
$$

for every $t \geq 0$ and $N \in \mathbb{N}$. The invariance principle states that, as $N$ goes to $\infty$, the finitedimensional distributions of $S_{\phi, H}^{N}$ converge to those of a Hermite process $W_{m, H}$ defined by (1.1) with a suitable constant $C(m, H)$. This is a remarkable property because it means that Hermite processes can be universal self-similar models in many applications of probability when long-range dependence arises. For instance, they have recently been used, in [10], to describe random media with long-range correlations for the study of wave propagation.

A limitation of Hermite processes and other fractional processes is the strong homogeneity of their properties as self-similarity, which are governed by the Hurst index $H$. In order to generalize fractional processes to less homogeneous processes, multifractional processes, as the class of multifractional Brownian motions [2], [13] for instance, have been introduced. Multifractional processes have locally, but not globally, the same properties as fractional processes. These properties are governed by a function $h$ substituting the constant $H$ in a suitable sense. For instance, multifractional processes satisfy the so-called local self-similarity property [2], [13].

As fractional Brownian motion and other Hermite processes, some nontrivial multifractional Gaussian processes satisfy invariance principles. From [4], we have the following result (see also [9] for a multidimensional version). Let $\left\{X_{j}(H)\right\}_{(j, H) \in \mathbb{N} \times(1 / 2,1)}$ be a Gaussian field satisfying some long-range assumptions related to (1.2): roughly speaking, there exists a continuous and symmetric function $R:\left(H_{1}, H_{2}\right) \rightarrow R\left(H_{1}, H_{2}\right)$ such that

$$
\mathbb{E}\left[X_{j}\left(H_{1}\right) X_{k}\left(H_{2}\right)\right] \sim R\left(H_{1}, H_{2}\right)|j-k|^{H_{1}+H_{2}-2} \quad \text { as }|j-k| \rightarrow \infty
$$

uniformly in $\left(H_{1}, H_{2}\right)$. Let $h$ be a continuous function taking its values in $\left(\frac{1}{2}, 1\right)$. We define the sequence of processes $\left\{S_{h}^{N}\right\}_{N \in \mathbb{N}}$ by

$$
S_{h}^{N}(t)=\sum_{j=1}^{\lfloor N t\rfloor} \frac{X_{j}(h(j / N))}{N^{h(j / N)}}
$$

for all $t \geq 0$ and $N \in \mathbb{N}$. Then, as $N$ goes to $\infty$, the finite-dimensional distributions of $S_{h}^{N}$ converge to those of a centered Gaussian process $S_{h}$ whose covariance is given for all $t$ and $s \geq 0$ by

$$
\mathbb{E}\left[S_{h}(t) S_{h}(s)\right]=\int_{0}^{t} \mathrm{~d} \theta \int_{0}^{s} \mathrm{~d} \sigma R(h(\theta), h(\sigma))|\theta-\sigma|^{h(\theta)+h(\sigma)-2},
$$


where $R$ is the continuous function derived from (1.4). The process $S_{h}$ is locally self-similar. If the function $h$ is constant then the process $S_{h}$ is a fractional Brownian motion. This result is then a generalization of the invariance principles presented above for $\phi=\mathrm{Id}: x \rightarrow x$. It also defines a class of multifractional Gaussian processes, which satisfies invariance principles.

In this work we generalize the invariance principles presented above. We study the asymptotic behavior of a sequence generalizing both (1.3) and (1.5). In particular, this sequence is defined by a Gaussian field $\left\{X_{j}(H)\right\}_{j, H}$ satisfying the long-range properties in (1.4), a function $\phi$ such that $\int_{\mathbb{R}} \phi(x)^{2} \mathrm{e}^{-x^{2} / 2} \mathrm{~d} x<\infty$ with Hermite rank equal to $m \in \mathbb{N}^{*}$, and a Hurst function $h$ taking its values in $\left(\frac{1}{2}, 1\right)$. We get as a limit a multifractional process $S_{m, h}$ that depends on the integer $m$ and the function $h$. If the function is a constant $H$ then the limit process is the Hermite process with Hurst index $H$ and Hermite order $m$. If the integer $m$ is equal to 1 then the limit process is a Gaussian multifractional process of the class obtained in [4]. Moreover, as Hermite processes, $S_{m, h}$ is Gaussian if and only if $m=1$. Our result then defines a class of multifractional processes, which can be Gaussian or non-Gaussian. Because these processes satisfy invariance principles, they can be suitable models when local self-similarity arises.

In contrast to what occurs in [4], the processes we study can be non-Gaussian. Hence, our work cannot be based only on second-order moments as in [4]. To prove our result, we use the convergence of multiple Wiener-Itô integrals and some properties of the Hermite polynomials. Moreover, to deal with multifractionality, we prove some uniform estimates to control the fluctuations of the Hurst index.

The paper is organized as follows. In Section 2 we recall some definitions and preliminary results about Hermite polynomials and multiple stochastic integrals, which are used throughout the paper. In Section 3 we establish the main results of the paper. Section 4 is devoted to the proofs.

\section{Preliminaries}

In this section we make precise some definitions and recall some results about Hermite polynomials and multiple stochastic integrals we use throughout this paper.

\subsection{Hermite polynomials}

For each positive integer $m \in \mathbb{N}$, the $m$ th Hermite polynomial $P_{m}$ is defined by

$$
P_{m}(x)=(-1)^{m} \mathrm{e}^{x^{2} / 2} \frac{\mathrm{d}^{m}}{\mathrm{~d} x^{m}} \mathrm{e}^{-x^{2} / 2}
$$

for every $x \in \mathbb{R}$. The family of the Hermite polynomials $\left\{P_{m}, m \in \mathbb{N}\right\}$ is an orthogonal basis of the space $L^{2}\left(\mathrm{e}^{-x^{2} / 2} \mathrm{~d} x\right)$ defined by

$$
L^{2}\left(\mathrm{e}^{-x^{2} / 2} \mathrm{~d} x\right)=\left\{\phi: \mathbb{R} \rightarrow \mathbb{R}, \phi \text { measurable and } \int_{\mathbb{R}}(\phi(x))^{2} \mathrm{e}^{-x^{2} / 2} \mathrm{~d} x<\infty\right\}
$$

endowed with the inner product $\langle\cdot, \cdot\rangle$ defined by

$$
\langle\cdot, \cdot\rangle:\left(\phi_{1}, \phi_{2}\right) \mapsto\left\langle\phi_{1}, \phi_{2}\right\rangle=\int_{\mathbb{R}} \phi_{1}(x) \phi_{2}(x) \mathrm{e}^{-x^{2} / 2} \frac{\mathrm{d} x}{\sqrt{2 \pi}} .
$$

The norm corresponding to $\langle\cdot, \cdot\rangle$ will be denoted by $\|\cdot\|$. For every (nonzero) function $\phi \in L^{2}\left(\mathrm{e}^{-x^{2} / 2} \mathrm{~d} x\right)$, there exists an integer $m_{\phi}$ such that $\left\langle\phi, P_{m_{\phi}}\right\rangle \neq 0$ and $\left\langle\phi, P_{m}\right\rangle=0$ for 
every $m=0, \ldots, m_{\phi}-1$. The integer $m_{\phi}$ is called the Hermite index of the function $\phi$. Hence, for every $\phi \in L^{2}\left(\mathrm{e}^{-x^{2} / 2} \mathrm{~d} x\right)$,

$$
\phi=\sum_{m=0}^{\infty} \frac{\left\langle\phi, P_{m}\right\rangle}{m !} P_{m}=\sum_{m=m_{\phi}}^{\infty} \frac{\left\langle\phi, P_{m}\right\rangle}{m !} P_{m},
$$

where the convergence of the series holds for the norm $\|\cdot\|$. If $X$ and $Y$ are two Gaussian random variables with mean 0 and variance 1 then, for all $j$ and $k$ in $\mathbb{N}^{*}$,

$$
\mathbb{E}\left[P_{j}(X) P_{k}(Y)\right]= \begin{cases}k !(\mathbb{E}[X Y])^{k} & \text { if } k=j, \\ 0 & \text { if } k \neq j\end{cases}
$$

As a consequence, for every $\phi \in L^{2}\left(\mathrm{e}^{-x^{2} / 2} \mathrm{~d} x\right)$ and every Gaussian random variable $X$ with mean 0 and variance 1 ,

$$
\mathbb{E}\left[|\phi(X)|^{2}\right]=\sum_{m=0}^{\infty} \frac{\left\langle\phi, P_{m}\right\rangle^{2}}{m !}<\infty .
$$

\subsection{Multiple Wiener-Itô integrals}

Many notions of multiple Wiener-Itô integrals [6], [8] with respect to Brownian motion have been introduced and have been used to define processes as Hermite processes [7], [17]. Here we have chosen to use the so-called multiple Wiener-Itô-Dobrushin integral defined in [6]. In this subsection we give a brief description of this integral, using its properties throughout the paper. We refer the reader to the seminal paper [6] for a complete construction and a detailed study.

For every $d \in \mathbb{N}^{*}$, we denote by $\hat{L}^{2}\left(\mathbb{R}^{d}\right)\left(\hat{L}^{2}(\mathbb{R})\right.$ when $\left.d=1\right)$ the space of square-integrable functions $f: \mathbb{R}^{d} \rightarrow \mathbb{C}$ satisfying, for every $\left(x_{1}, \ldots, x_{d}\right) \in \mathbb{R}^{d}$,

$$
f\left(x_{1}, \ldots, x_{d}\right)=\overline{f\left(-x_{1}, \ldots,-x_{d}\right)},
$$

and, for every permutation $\sigma$ on $\{1, \ldots, d\}$,

$$
f\left(x_{1}, \ldots, x_{d}\right)=f\left(x_{\sigma(1)}, \ldots, x_{\sigma(d)}\right) .
$$

Let $B$ be a real Brownian motion. We define the random measure $\hat{B}$ by

$$
\hat{B}(\psi):=\int_{\mathbb{R}} \hat{\psi}(\xi) \mathrm{d} B_{\xi}
$$

for every $\psi \in \hat{L}^{2}(\mathbb{R})$, where $\hat{\psi}$ is the Fourier transform of $\psi$ and the integral of the right-hand side is the classical Wiener-Itô integral in dimension one. We also denote by $\int_{\mathbb{R}} \psi(x) \mathrm{d} \hat{B}_{x}$ the random variable $\hat{B}(\psi)$. Because $\psi \in \hat{L}^{2}(\mathbb{R}), \hat{B}(\psi)$ is a real Gaussian variable with mean 0 and variance $\int_{\mathbb{R}}|\psi(x)|^{2} \mathrm{~d} x$. Let $d \in \mathbb{N}^{*}$, and consider a function $f \in \hat{L}^{2}\left(\mathbb{R}^{d}\right)$. A multiple random integral of $f$ is defined in [6] from $\hat{B}$ by an approximation of $f$ with step functions in $\hat{L}^{2}\left(\mathbb{R}^{d}\right)$. This is the so-called Wiener-Itô-Dobrushin integral of $f$, which we denote in this paper by $\int_{\mathbb{R}^{d}} f \mathrm{~d} \hat{B}^{\otimes d}$ or

$$
\int_{\mathbb{R}^{d}} f\left(x_{1}, \ldots, x_{d}\right) \mathrm{d} \hat{B}_{x_{1}} \cdots \mathrm{d} \hat{B}_{x_{d}} .
$$


It is a real random variable with mean 0 and variance

$$
\mathbb{E}\left[\left(\int_{\mathbb{R}^{d}} f \mathrm{~d} \hat{B}^{\otimes d}\right)^{2}\right]=d ! \int_{\mathbb{R}^{d}}\left|f\left(x_{1}, \ldots, x_{d}\right)\right|^{2} \mathrm{~d} x_{1} \cdots \mathrm{d} x_{d}
$$

and is Gaussian if and only if $d=1$. The following Fubini-type formula is one of the most important properties of this integral [6].

Lemma 2.1. Let $P_{d}$ be the Hermite polynomial of rank $d$. For every $\psi \in \hat{L}^{2}(\mathbb{R})$ satisfying $\int_{\mathbb{R}}|\psi(\xi)|^{2} \mathrm{~d} \xi=1$

$$
P_{d}\left(\int_{\mathbb{R}} \psi(x) \mathrm{d} \hat{B}_{x}\right)=\int_{\mathbb{R}^{d}} \psi\left(x_{1}\right) \cdots \psi\left(x_{d}\right) \mathrm{d} \hat{B}_{x_{1}} \cdots \mathrm{d} \hat{B}_{x_{d}} .
$$

The following lemma states a change-of-variable formula. It is due to the self-similarity of Brownian motion [6].

Lemma 2.2. For every $\varepsilon>0$, we have the equality in distribution

$$
\int_{\mathbb{R}^{d}} f\left(x_{1}, \ldots, x_{d}\right) \mathrm{d} \hat{B}_{x_{1}} \cdots \mathrm{d} \hat{B}_{x_{d}} \stackrel{\mathrm{D}}{=} \varepsilon^{m / 2} \int_{\mathbb{R}^{d}} f\left(\varepsilon x_{1}, \ldots, \varepsilon x_{d}\right) \mathrm{d} \hat{B}_{x_{1}} \cdots \mathrm{d} \hat{B}_{x_{d}} .
$$

The following lemma gives another change-of-variable formula. It is a direct consequence of Proposition 4.2 of [6].

Lemma 2.3. Let $z: \mathbb{R} \rightarrow \mathbb{C}$ be a bounded and measurable function satisfying $z(x)=\overline{z(-x)}$ and $|z(x)|=1$ for every $x \in \mathbb{R}$. Then, we have the equality in distribution

$$
\int_{\mathbb{R}^{d}} f\left(x_{1}, \ldots, x_{d}\right) \mathrm{d} \hat{B}_{x_{1}} \cdots \mathrm{d} \hat{B}_{x_{d}} \stackrel{\mathrm{D}}{=} \int_{\mathbb{R}^{d}} f\left(x_{1}, \ldots, x_{d}\right) z\left(x_{1}\right) \cdots z\left(x_{d}\right) \mathrm{d} \hat{B}_{x_{1}} \cdots \mathrm{d} \hat{B}_{x_{d}} .
$$

By using the linearity of the integral, (2.4), and the bounded convergence theorem, we can prove the following lemma.

Lemma 2.4. Let $\left\{f_{N}\right\}_{N \in \mathbb{N}}$ be a sequence of functions in $\hat{L}^{2}\left(\mathbb{R}^{d}\right)$. We assume that there exist two functions $f$ and $f^{*}$ in $\hat{L}^{2}\left(\mathbb{R}^{d}\right)$ such that, for almost every $x \in \mathbb{R}^{d}, \lim _{N \rightarrow \infty} f_{N}(x)=f(x)$ and $\sup _{N}\left|f_{N}(x)\right| \leq f^{*}(x)$. Then

$$
\lim _{N \rightarrow \infty} \mathbb{E}\left[\left(\int_{\mathbb{R}^{d}} f_{N} \mathrm{~d} \hat{B}^{\otimes d}-\int_{\mathbb{R}^{d}} f \mathrm{~d} \hat{B}^{\otimes d}\right)^{2}\right]=0 .
$$

Finally, we can generalize (2.4) to every moment by using hypercontractivity arguments (see, for instance, [11] or [12]).

Lemma 2.5. Let $p \in \mathbb{N}^{*}$. There exists a constant $C=C(d, p)>0$ such that, for every $f \in \hat{L}^{2}\left(\mathbb{R}^{d}\right)$,

$$
\mathbb{E}\left[\left(\int_{\mathbb{R}^{d}} f \mathrm{~d} \hat{B}^{\otimes d}\right)^{2 p}\right] \leq C(d, p)\left(\int_{\mathbb{R}^{d}}\left|f\left(x_{1}, \ldots, x_{d}\right)\right|^{2} \mathrm{~d} x_{1} \cdots \mathrm{d} x_{d}\right)^{p} .
$$




\section{Main results}

We fix $m \in \mathbb{N}^{*}$, and define

$$
b=1-\frac{1}{2 m}
$$

We consider the Gaussian field $X=\left\{X_{n}(H)\right\}_{(n, H) \in \mathbb{N} \times(b, 1)}$ defined by

$$
X_{n}(H)=\int_{-a}^{a} \exp (\mathrm{i} n x) g(H, x)|x|^{1 / 2-H} \mathrm{~d} \hat{B}_{x}
$$

for all $n \in \mathbb{N}$ and $H \in(b, 1)$, where $a \in(0,2 \pi / m), \hat{B}$ is the Fourier transform of the random Brownian measure, and $g:(b, 1) \times(-a, a) \rightarrow \mathbb{C}$ is a measurable function. The right-hand side of (3.1) is a stochastic integral defined as in Section 2.2. We assume that the function $g$ satisfies the following properties.

- For every $(H, x) \in(b, 1) \times(0, a), g(H, x)=\overline{g(H,-x)}$. This property ensures that the field $X$ is real.

- For every $H \in(b, 1)$,

$$
\left.\int_{-a}^{a}|g(H, x)|^{2}|x|\right|^{1-2 H} \mathrm{~d} x=1
$$

so that $\mathbb{E}\left[X_{n}(H)^{2}\right]=1$.

- The function $g$ is twice continuously differentiable on $(b, 1) \times(-a, a)$. Then, for every $(H, x) \in(b, 1) \times(-a, a)$, we define

$$
g_{0}(H)=g(H, 0) \quad \text { and } \quad g_{1}(H, x)=\int_{0}^{x} \frac{\partial g}{\partial \xi}(H, \xi) \mathrm{d} \xi,
$$

so that $g=g_{0}+g_{1}$ and

$$
\lim _{x \rightarrow 0} \sup _{H \in K}\left(\left|g_{1}(H, x)\right|+\left|\frac{\partial g_{1}}{\partial H}(H, x)\right|\right)=0
$$

for every compact set $K$ of $(b, 1)$.

The assumptions above ensure that the covariance function satisfies the uniform long-range property of [4]. More precisely, Lemma A.1 (see Appendix A) states that, for every compact set $K \subset(b, 1)$,

$$
\lim _{j-k \rightarrow \infty} \sup _{\left(H_{1}, H_{2}\right) \in K^{2}}\left|(j-k)^{2-H_{1}-H_{2}} \mathbb{E}\left[X_{j}\left(H_{1}\right) X_{k}\left(H_{2}\right)\right]-R\left(H_{1}, H_{2}\right)\right|=0,
$$

where

$$
R\left(H_{1}, H_{2}\right)=g_{0}\left(H_{1}\right) g_{0}\left(H_{2}\right) \int_{\mathbb{R}} \exp (\mathrm{i} x)|x|^{1-H_{1}-H_{2}} \mathrm{~d} x
$$

for every $\left(H_{1}, H_{2}\right) \in(b, 1)^{2}$.

We consider a function $\phi \in L^{2}\left(\mathrm{e}^{-x^{2} / 2} \mathrm{~d} x\right)$ with Hermite rank equal to $m \in \mathbb{N}^{*}$. Note that $\phi$ then satisfies the centering condition

$$
\int_{\mathbb{R}} \phi(x) \mathrm{e}^{-x^{2} / 2} \mathrm{~d} x=0
$$


We consider a continuously differentiable function $h:[0, \infty) \rightarrow\left(\frac{1}{2}, 1\right)$ and set

$$
\tilde{h}:=1+\frac{h-1}{m}:[0, \infty) \rightarrow(b, 1) .
$$

For all $t \geq 0$ and $N \in \mathbb{N}^{*}$, we define

$$
S_{\phi, h}^{N}(t):=\sum_{j=1}^{\lfloor N t\rfloor} \frac{\phi\left(X_{j}\left(h_{j}^{N}\right)\right)}{N^{h(j / N)}}
$$

where, for every $j \in\{1, \ldots,\lfloor N t\rfloor\}$,

$$
h_{j}^{N}=1+\frac{h(j / N)-1}{m}=\tilde{h}\left(\frac{j}{N}\right) .
$$

For every $\left(x_{1}, \ldots, x_{m}, t\right) \in\left(\mathbb{R}^{*}\right)^{m} \times[0, \infty)$, we set

$$
f_{m, h}\left(x_{1}, \ldots, x_{m}, t\right)=\int_{0}^{t} \exp \left(\mathrm{i} \theta \sum_{l=1}^{m} x_{l}\right) \tilde{g}(\theta)\left|x_{1} \cdots x_{m}\right|^{1 / 2-\tilde{h}(\theta)} \mathrm{d} \theta,
$$

where

$$
\tilde{g}=\frac{\left\langle\phi, P_{m}\right\rangle}{m !}\left(g_{0} \circ \tilde{h}\right)^{m}
$$

For every $t \geq 0$, we define

$$
S_{m, h}(t)=\int_{\mathbb{R}^{m}} f_{m, h}\left(x_{1}, \ldots, x_{m}, t\right) \mathrm{d} \hat{B}_{x_{1}} \cdots \mathrm{d} \hat{B}_{x_{m}} .
$$

For all $t, u \geq 0$ and $\varepsilon \in(0,1)$, we set

$$
T_{m, h, t}^{\varepsilon}(u)=\frac{S_{m, h}(t+\varepsilon u)-S_{m, h}(t)}{\varepsilon^{h(t)}}
$$

and

$$
T_{m, h, t}(u)=\tilde{g}(t) \int_{\mathbb{R}^{m}} \frac{\exp \left(\mathrm{i} u \sum_{l=1}^{m} x_{l}\right)-1}{\mathrm{i}\left(\sum_{l=1}^{m} x_{l}\right)\left|x_{1} \cdots x_{m}\right|^{\tilde{h}(t)-1 / 2}} \mathrm{~d} \hat{B}_{x_{1}} \cdots \mathrm{d} \hat{B}_{x_{m}} .
$$

For any real interval $I, \mathscr{D}(I)$ is the space of càdlàg functions on $I$ with the Skorokhod topology (see [3, Chapter 3]) and $\mathcal{C}(I)$ is the space of continuous functions on $I$ with the uniform topology on each compact set. For a real continuous function $w$ and a point $t$ in the domain of definition of $w$, the local Hölder exponent of $w$ around $t$ is denoted by $\alpha_{w}(t)$ and defined by

$$
\alpha_{w}(t)=\sup \left\{\alpha \in(0,1]: \lim _{s \rightarrow t} \frac{|w(t)-w(s)|}{|t-s|^{\alpha}}<\infty\right\} .
$$

The two main results of this paper can now be stated. The first result concerns an invariance principle.

Theorem 3.1. The process $S_{m, h}=\left\{S_{m, h}(t)\right\}_{t \geq 0}$ is continuous (up to a modification) and $S_{\phi, h}^{N}=\left\{S_{\phi, h}^{N}(t)\right\}_{t \geq 0}$ converges in distribution to $S_{m, h}$ in $\mathscr{D}([0, \infty))$ as $N \rightarrow \infty$. 
The second main result deals with sample path properties (local self-similarity and local Hölder exponent) of the limit process $S_{m, h}$.

Theorem 3.2. Let $t \geq 0$. The process $T_{m, h, t}^{\varepsilon}=\left\{T_{m, h, t}^{\varepsilon}(u)\right\}_{u \geq 0}$ converges in distribution to $T_{m, h, t}=\left\{T_{m, h, t}(u)\right\}_{u \geq 0}$ in $\mathrm{C}([0, \infty))$ as $\varepsilon \rightarrow 0$. Moreover, the local Hölder exponent of $S_{m, h}$ around $t$ is equal to $h(t)$.

Theorems 3.1 and 3.2 establish that sequences of processes defined as in (3.5), in particular from a function $\phi$ in $L^{2}\left(\mathrm{e}^{-x^{2} / 2} \mathrm{~d} x\right)$ of Hermite rank $m$ and a Hurst function $h$, converge to a multifractional process $S_{m, h}$ with Hurst function $h$ and represented as a multiple integral of order $m$. Therefore, because the process $S_{m, h}$ is defined as the limit of an invariance principle, it can be a universal model when local self-similarity and long-range dependence arise in a Gaussian or non-Gaussian framework.

Theorem 3.1 generalizes the results of [7] and [17] to a multifractional setting. Indeed, if we assume that $h \equiv H \in\left(\frac{1}{2}, 1\right)$ then Theorem 3.1 is the main result of [7] and [17]. In particular, the limit process $S_{m, H}$ can be written as $W_{m, H}$ in (1.1) with the constant

$$
C(m, H)=\frac{\left\langle\phi, P_{m}\right\rangle}{m !}\left(g_{0}(\tilde{H})\right)^{m}=\frac{\left\langle\phi, P_{m}\right\rangle}{m !}\left(\frac{R(\tilde{H}, \tilde{H})}{\int_{\mathbb{R}} \mathrm{e}^{\mathrm{i} \xi}|\xi|^{1-2 \tilde{H}} \mathrm{~d} \xi}\right)^{m / 2},
$$

where

$$
\tilde{H}:=1+\frac{H-1}{m} \in(b, 1) .
$$

Theorem 3.1 is also an extension of the main result of [4] (Theorem 2) to a non-Gaussian framework. Indeed, if we assume that $\phi=\mathrm{Id}: x \rightarrow x$ then $m=1$ and the limit process is $S_{1, h}$, which is a centered Gaussian process of covariance

$$
(t, s) \mapsto \mathbb{E}\left[S_{1, h}(t) S_{1, h}(s)\right]=\int_{0}^{t} \mathrm{~d} \theta \int_{0}^{s} \mathrm{~d} \sigma R(h(\theta), h(\sigma))|\theta-\sigma|^{h(\theta)+h(\sigma)-2}
$$

with $R$ defined by (3.4).

To conclude this section, let us note the connection between our work and [15]. Let $Y_{h}=$ $\left\{Y_{h}(t)\right\}_{t \geq 0}$ be the process defined for every $t \geq 0$ by

$$
Y_{h}(t)=\mathcal{R} \int_{\mathbb{R}}\left(\int_{0}^{t} \frac{\exp (\mathrm{i} y \theta)}{|y|^{h(\theta)-1 / 2}} \mathrm{~d} \theta\right) \mathrm{d} \tilde{B}(y),
$$

where $\mathrm{d} \tilde{B}$ is a complex Gaussian measure and $\mathcal{R}$ stands for the real part. This process is called integrated fractional white noise and has been introduced in [15] as an alternative to multifractional Brownian motion. An advantage is the fact that it is a multifractional Gaussian process without undesirable oscillations that multifractional Brownian motion has (see [15]). Note that if we let $g_{0} \equiv\left(m ! /\left\langle\phi, P_{m}\right\rangle\right)^{1 / m}$ then, for $m=1$, the process $S_{1, h}$ has the same distributions as $Y_{h}$. Therefore, Theorem 3.1 states that $Y_{h}$ is the limit of an invariance principle. This is another interest of $Y_{h}$. Moreover, for every $m \geq 2$, the process $S_{m, h}$ is a natural generalization of $Y_{h}$ to a non-Gaussian framework.

\section{Proofs}

In this section we prove Theorems 3.1 and 3.2 and we proceed as follows. In Subsection 4.1 we establish a technical lemma. We prove the convergence of the finite-dimensional 
distributions of $S_{\phi, h}^{N}$ in Subsection 4.2. The regularity properties of $S_{m, h}$ are established in Subsection 4.3. Finally, we deal with the tightness for the Skorokhod topology in Subsection 4.4. Throughout this section, for every set $E$ and every subset $A \subset E$, we denote by $1_{A}$ the function defined on $E$ such that $1_{A}(a)=1$ if $a \in A$ and $1_{A}(a)=0$ if $a \in E-A$.

\subsection{Technical lemma}

In the following lemma we prove, for every $T>0$, the existence of a function $\tilde{f}_{T}$ that is useful in the sequel to establish uniform bounds.

Lemma 4.1. For every $T>0$, there exists a function $\tilde{f}_{T} \in L^{2}\left(\mathbb{R}^{m}, \mathbb{R}\right)$ such that, for every $t \in[0, T]$, every $H \in[\min \tilde{h}, \max \tilde{h}]$, and almost every $\left(x_{1}, \ldots, x_{m}\right) \in \mathbb{R}^{m}$,

$$
\left|\frac{\exp \left(\mathrm{i} t \sum_{l=1}^{m} x_{l}\right)-1}{\left|x_{1} \cdots x_{m}\right|^{H-1 / 2} \sum_{l=1}^{m} x_{l}}\right|\left(1+|\ln | x_{1} \cdots x_{m}||\right) \leq \tilde{f}_{T}(x) .
$$

Proof. We fix $T>0$. We define $L\left(x_{1}, \ldots, x_{m}\right)=\left(1+|\ln | x_{1} \cdots x_{m}||\right)^{2}$ and

$$
\begin{aligned}
& \tilde{f}_{T}\left(x_{1}, \ldots, x_{m}\right) \\
& \quad=\sqrt{\sum_{H=\min \tilde{h}, \max \tilde{h}} \frac{T^{2} 1_{\left\{\left|\sum_{l=1}^{m} x_{l}\right| \leq 1\right\}}+4\left|\sum_{l=1}^{m} x_{l}\right|^{-2} 1_{\left\{\left|\sum_{l=1}^{m} x_{l}\right|>1\right\}}}{\left|x_{1} \cdots x_{m}\right|^{2 H-1}} L\left(x_{1}, \ldots, x_{m}\right) .} .
\end{aligned}
$$

We have

$$
\max _{H \in[\min \tilde{h}, \max \tilde{h}]} \frac{1}{\left|x_{1} \cdots x_{m}\right|^{2 H-1}} \leq \frac{1}{\left|x_{1} \cdots x_{m}\right|^{2 \min \tilde{h}-1}}+\frac{1}{\left|x_{1} \cdots x_{m}\right|^{2 \max \tilde{h}-1}} .
$$

Then, for every $t \in[0, T]$,

$$
\left|\frac{\exp \left(\mathrm{i} t \sum_{l=1}^{m} x_{l}\right)-1}{\sum_{l=1}^{m} x_{l}}\right|^{2} \max _{H \in[\min \tilde{h}, \max \tilde{h}]} \frac{L\left(x_{1}, \ldots, x_{m}\right)}{\left|x_{1} \cdots x_{m}\right|^{2 H-1}} \leq \tilde{f}_{T}\left(x_{1}, \ldots, x_{m}\right)^{2} .
$$

It is then enough to prove that, for $H \in\{\min \tilde{h}, \max \tilde{h}\}$, the function

$$
\left(x_{1}, \ldots, x_{m}\right) \mapsto \frac{T^{2} 1_{\left\{\left|\sum_{l=1}^{m} x_{l}\right| \leq 1\right\}}+4\left|\sum_{l=1}^{m} x_{l}\right|^{-2} 1_{\left\{\left|\sum_{l=1}^{m} x_{l}\right|>1\right\}}}{\left|x_{1} \cdots x_{m}\right|^{2 H-1}} L\left(x_{1}, \ldots, x_{m}\right)
$$

is integrable. We successively make the substitutions $y_{j}=x_{1}+\cdots+x_{j}$ for every $j \in\{1, \ldots, m\}, z_{k}=y_{k} / y_{k+1}$ for every $k \in\{1, \ldots, m-1\}$, and $z_{m}=y_{m}$ to get

$$
\begin{aligned}
\int_{\mathbb{R}^{m}} & \frac{T^{2} 1_{\left\{\left|\sum_{l=1}^{m} x_{l}\right| \leq 1\right\}}+4\left|\sum_{l=1}^{m} x_{l}\right|^{-2} 1_{\left\{\left|\sum_{l=1}^{m} x_{l}\right|>1\right\}}}{\left|x_{1} \cdots x_{m}\right|^{2 H-1}} L\left(x_{1}, \ldots, x_{m}\right) \mathrm{d} x_{1} \cdots \mathrm{d} x_{m} \\
= & \int_{\mathbb{R}^{m}} \frac{T^{2} 1_{\left\{\left|y_{m}\right| \leq 1\right\}}+4\left|y_{m}\right|^{-2} 1_{\left\{\left|y_{m}\right|>1\right\}}}{\left|y_{1}\left(y_{2}-y_{1}\right) \cdots\left(y_{m}-y_{m-1}\right)\right|^{2 H-1}} L\left(y_{1}, y_{2}-y_{1}, \ldots, y_{m}-y_{m-1}\right) \mathrm{d} y_{1} \cdots \mathrm{d} y_{m} \\
= & \int_{\mathbb{R}} \frac{T^{2} 1_{\left\{\left|z_{m}\right| \leq 1\right\}}+4\left|z_{m}\right|^{-2} 1_{\left\{\left|z_{m}\right|>1\right\}}}{\left|z_{m}\right|^{2 m(H-1)+1}} \mathrm{~d} z_{m} \int_{\mathbb{R}} \frac{\mathrm{d} z_{m-1}}{\left|1-z_{m-1}\right|^{2 H-1}\left|z_{m-1}\right|^{2(m-1)(H-1)+1}} \times \cdots \\
& \times \int_{\mathbb{R}} \frac{\mathrm{d} z_{1}}{\left|1-z_{1}\right|^{2 H-1}\left|z_{1}\right|^{2 H-1}} L\left(\prod_{k=1}^{m} z_{k},\left(1-z_{1}\right) \prod_{k=2}^{m} z_{k}, \ldots,\left(1-z_{m-1}\right) z_{m}\right) .
\end{aligned}
$$


The right-hand side above can be bounded by a finite sum of terms of the form

$$
\begin{aligned}
& \int_{\mathbb{R}} \frac{T^{2} 1_{\left\{\left|z_{m}\right| \leq 1\right\}}+4\left|z_{m}\right|^{-2} 1_{\left\{\left|z_{m}\right|>1\right\}}}{\left|z_{m}\right|^{2 m(H-1)+1}} \mathrm{~d} z_{m} \int_{\mathbb{R}} \frac{\mathrm{d} z_{m-1}}{\left|1-z_{m-1}\right|^{2 H-1}\left|z_{m-1}\right|^{2(m-1)(H-1)+1}} \times \cdots \\
& \times\left.\int_{\mathbb{R}} \frac{\mathrm{d} z_{1}}{\left|1-z_{1}\right|^{2 H-1}\left|z_{1}\right|^{2 H-1}}|\ln | z_{k}\right|^{\mu}|\ln | 1-z_{j} \|^{\nu},
\end{aligned}
$$

where $k, j, \mu$, and $v$ are integers. The terms of the form (4.1) are finite since $H \in(1-1 /(2 m), 1)$ and by Bertrand's test. This concludes the proof.

\subsection{Convergence of the finite-dimensional distributions}

First, we deal with the sequence $\left\{S_{P_{m}, h}^{N}\right\}_{N \in \mathbb{N}}$ defined by

$$
S_{P_{m}, h}^{N}(t)=\sum_{j=1}^{\lfloor N t\rfloor} \frac{P_{m}\left(X_{j}\left(h_{j}^{N}\right)\right)}{N^{h(j / N)}}
$$

for all $t \geq 0$ and $N \in \mathbb{N}$. From now on, we denote by $\mathrm{d} \hat{B}_{x}^{\otimes m}$ the product $\prod_{l=1}^{m} \mathrm{~d} \hat{B}_{x_{l}}$ when $x=\left(x_{1}, \ldots, x_{d}\right)$.

Lemma 4.2. For every $N \in \mathbb{N}$, the process $S_{P_{m}, h}^{N}$ is equal in distribution to the process $\tilde{S}_{m, h}^{N}$ defined by

$$
\tilde{S}_{m, h}^{N}(t)=\int_{(-N a, N a)^{m}} \mathrm{~d} \hat{B}_{x}^{\otimes m} \frac{1}{N} \sum_{j=1}^{\lfloor N t\rfloor} \prod_{l=1}^{m} \exp \left(\frac{\mathrm{i} j x_{l}}{N}\right) g\left(h_{j}^{N}, \frac{x_{l}}{N}\right)\left|x_{l}\right|^{1 / 2-h_{j}^{N}}
$$

for every $t \geq 0$.

Proof. Using (2.5), we obtain

$$
P_{m}\left(X_{j}\left(h_{j}^{N}\right)\right)=\int_{(-a, a)^{m}} \prod_{l=1}^{m} \exp \left(\mathrm{i} j x_{l}\right) g\left(h_{j}^{N}, x_{l}\right)\left|x_{l}\right|^{1 / 2-h_{j}^{N}} \mathrm{~d} \hat{B}_{x_{l}}
$$

almost surely. We then have

$$
S_{P_{m}, h}^{N}(t)=\sum_{j=1}^{\lfloor N t\rfloor} \frac{1}{N^{1-m / 2}} \int_{(-a, a)^{m}} \mathrm{~d} \hat{B}_{x}^{\otimes m} \prod_{l=1}^{m} \exp \left(\mathrm{i} j x_{l}\right) g\left(h_{j}^{N}, x_{l}\right)\left|N x_{l}\right|^{1 / 2-h_{j}^{N}} .
$$

Making the substitution $x \rightarrow x / N$ and using (2.6), we obtain

$$
S_{P_{m}, h}^{N} \stackrel{\mathrm{D}}{=} t \mapsto \sum_{j=1}^{\lfloor N t\rfloor} \frac{1}{N} \int_{(-N a, N a)^{m}} \mathrm{~d} \hat{B}_{x}^{\otimes m} \prod_{l=1}^{m} \exp \left(\frac{\mathrm{i} j x_{l}}{N}\right) g\left(h_{j}^{N}, \frac{x_{l}}{N}\right)\left|x_{l}\right|^{1 / 2-h_{j}^{N}} .
$$

This concludes the proof by the linearity of the multiple integral.

We now aim to prove the convergence of $\left\{\tilde{S}_{m, h}^{N}(t)\right\}_{N \in \mathbb{N}}$ in $L^{2}(\Omega, \mathbb{R})$ for every $t$. We introduce the sequence of functions $\left\{f^{N}\right\}_{N \in \mathbb{N}}$ defined by

$$
\begin{aligned}
f^{N}:[0, \infty) \times \mathbb{R}^{m} & \rightarrow \mathbb{C}, \\
(t, x) & \mapsto 1_{(-N a, N a)^{m}}(x) \frac{1}{N} \sum_{j=1}^{\lfloor N t\rfloor} \prod_{l=1}^{m} \exp \left(\frac{\mathrm{i} j x_{l}}{N}\right) \frac{g\left(h_{j}^{N}, x_{l} / N\right)}{\left|x_{l}\right|^{h_{j}^{N}-1 / 2}},
\end{aligned}
$$

and we establish the following lemma. 
Lemma 4.3. For every $t \geq 0$, there exists a function $f_{t}^{*} \in L^{2}\left(\mathbb{R}^{m}, \mathbb{R}\right)$ such that, for all $x \in \mathbb{R}^{m}$ and $N \in \mathbb{N}$,

$$
\left|f^{N}(t, x)\right| \leq f_{t}^{*}(x)
$$

Proof. We have

$$
\begin{aligned}
f^{N}(t, x)= & 1_{(-N a, N a)}(x) \frac{\mathrm{i} \sum_{l=1}^{m} x_{l} / N}{1-\exp \left(-\mathrm{i} \sum_{l=1}^{m} x_{l} / N\right)} \\
& \times \sum_{j=1}^{\lfloor N t\rfloor} \frac{\exp \left(\mathrm{i} j \sum_{l=1}^{m} x_{l} / N\right)-\exp \left(\mathrm{i}(j-1) \sum_{l=1}^{m} x_{l} / N\right)}{\mathrm{i} \sum_{l=1}^{m} x_{l}} G_{j}^{N}(x),
\end{aligned}
$$

where

$$
G_{j}^{N}(x)=\prod_{l=1}^{m} \frac{g\left(h_{j}^{N}, x_{l} / N\right)}{\left|x_{l}\right|^{h_{j}^{N}-1 / 2}} .
$$

We write

$$
f^{N}(t, x)=f^{N, 1}(t, x)-f^{N, 2}(t, x)
$$

with

$$
\begin{aligned}
f^{N, 1}(t, x)= & 1_{(-N a, N a)}(x) \frac{\mathrm{i} \sum_{l=1}^{m} x_{l} / N}{1-\exp \left(-\mathrm{i} \sum_{l=1}^{m} x_{l} / N\right)} \\
\times & \sum_{j=1}^{\lfloor N t\rfloor} \frac{1}{\mathrm{i} \sum_{l=1}^{m} x_{l}}\left(G_{j}^{N}(x)\left(\exp \left(\mathrm{i} j \sum_{l=1}^{m} \frac{x_{l}}{N}\right)-1\right)\right. \\
& \left.-G_{j-1}^{N}(x)\left(\exp \left(\mathrm{i}(j-1) \sum_{l=1}^{m} \frac{x_{l}}{N}\right)-1\right)\right) \\
= & 1_{(-N a, N a)}(x) \frac{\mathrm{i} \sum_{l=1}^{m} x_{l} / N}{1-\exp \left(-\mathrm{i} \sum_{l=1}^{m} x_{l} / N\right)} G_{\lfloor N t\rfloor}^{N}(x) \frac{\exp \left(\mathrm{i}\lfloor N t\rfloor \sum_{l=1}^{m} x_{l} / N\right)-1}{\mathrm{i} \sum_{l=1}^{m} x_{l}}
\end{aligned}
$$

and

$$
\begin{aligned}
f^{N, 2}(t, x)= & 1_{(-N a, N a)}(x) \frac{\mathrm{i} \sum_{l=1}^{m} x_{l} / N}{1-\exp \left(-\mathrm{i} \sum_{l=1}^{m} x_{l} / N\right)} \\
& \times \sum_{j=1}^{\lfloor N t\rfloor} \frac{\exp \left(\mathrm{i}(j-1) \sum_{l=1}^{m} x_{l} / N\right)-1}{\mathrm{i} \sum_{l=1}^{m} x_{l}}\left(G_{j}^{N}(x)-G_{j-1}^{N}(x)\right) .
\end{aligned}
$$

First, we deal with $f^{N, 1}$. Because $g$ is bounded, there exists $M_{1}>0$ such that, for every $N$ and almost every $x$,

$$
\left|f^{N, 1}(t, x)\right| \leq M_{1}\left|\frac{\exp \left(\mathrm{i}\lfloor N t\rfloor \sum_{l=1}^{m} x_{l} / N\right)-1}{\left|x_{1} \cdots x_{m}\right|^{h_{\lfloor N t\rfloor}^{N}-1 / 2} \sum_{l=1}^{m} x_{l}}\right| .
$$

Then, by Lemma 4.1, there exists a function $\tilde{f}_{t, 1} \in L^{2}\left(\mathbb{R}^{m}, \mathbb{R}\right)$ such that

$$
\left|\frac{\exp \left(\mathrm{i}\lfloor N t\rfloor \sum_{l=1}^{m} x_{l} / N\right)-1}{\left|x_{1} \cdots x_{m}\right|^{h^{N}}-1 / 2 \sum_{l=1}^{m} x_{l}}\right| \leq \tilde{f}_{t, 1}(x),
$$


so that we get

$$
\left|f^{N, 1}(t, x)\right| \leq M_{1} \tilde{f}_{t, 1}(x) .
$$

We now deal with $f^{N, 2}$. By Taylor's formula we obtain

$$
\begin{aligned}
\left|G_{j}^{N}(x)-G_{j-1}^{N}(x)\right| & \\
\leq \frac{\max \left|\tilde{h}^{\prime}\right|}{N} \max _{H \in[\min \tilde{h}, \max \tilde{h}]} & \frac{-\ln \left|x_{1} \cdots x_{m}\right|}{\left|x_{1} \cdots x_{m}\right|^{H-1 / 2}} \prod_{l=1}^{m} g\left(H, \frac{x_{l}}{N}\right) \\
& +\frac{1}{\left|x_{1} \cdots x_{m}\right|^{H-1 / 2}} \sum_{k=1}^{m}\left(\frac{\partial g}{\partial H}\left(H, \frac{x_{k}}{N}\right)\right) \prod_{l=1, l \neq k}^{m} g\left(H, \frac{x_{l}}{N}\right) \mid .
\end{aligned}
$$

Since $g$ and $\partial g / \partial H$ are bounded, there exists a constant $M_{2}>0$, which depends only on $h$ and $g$, such that, for almost every $x$ and every $N$,

$$
\left|f^{N, 2}(t, x)\right| \leq \frac{M_{2}}{N} \sum_{j=1}^{\lfloor N t\rfloor} \frac{\left|\exp \left(\mathrm{i}(j-1) \sum_{l=1}^{m} x_{l} / N\right)-1\right|}{\left|\sum_{l=1}^{m} x_{l}\right|} \max _{H \in[\min \tilde{h}, \max \tilde{h}]} \frac{1+|\ln | x_{1} \cdots x_{m}||}{\left|x_{1} \cdots x_{m}\right|^{H-1 / 2}} .
$$

As in the case of $f^{N, 1}$, by Lemma 4.1 , there exists a function $\tilde{f}_{t, 2} \in L^{2}\left(\mathbb{R}^{m}, \mathbb{R}\right)$ such that, for all $N$ and $j$ and almost every $x$,

$$
\left|\frac{\exp \left(\mathrm{i}(j-1) \sum_{l=1}^{m} x_{l} / N\right)-1}{\sum_{l=1}^{m} x_{l}}\right| \max _{H \in[\min \tilde{h}, \max \tilde{h}]} \frac{1+|\ln | x_{1} \cdots x_{m}||}{\left|x_{1} \cdots x_{m}\right|^{H-1 / 2}} \leq \tilde{f}_{t, 2}(x) .
$$

We then get

$$
\left|f^{N, 2}(t, x)\right| \leq M_{2} \tilde{f}_{t, 2}(x) .
$$

Hence, taking $\tilde{f}_{t}=M_{1} \tilde{f}_{t, 1}+M_{2} \tilde{f}_{t, 2}$ and combining (4.2) and (4.3) completes the proof.

The convergence of $\left\{\tilde{S}_{m, h}^{N}\right\}_{n \in \mathbb{N}}$ can now be established.

Lemma 4.4. For every $t \geq 0$, as $N \rightarrow \infty, \tilde{S}_{m, h}^{N}(t)$ converges in $L^{2}(\Omega, \mathbb{R})$ to $\tilde{S}_{m, h}^{\infty}(t)$ given by

$$
\tilde{S}_{m, h}^{\infty}(t)=\int_{\mathbb{R}^{m}} \mathrm{~d} \hat{B}_{x}^{\otimes m} \int_{0}^{t} \exp \left(\mathrm{i} \theta \sum_{l=1}^{m} x_{l}\right) g_{0}(\tilde{h}(\theta))^{m}\left|x_{1} \cdots x_{m}\right|^{1 / 2-\tilde{h}(\theta)} \mathrm{d} \theta .
$$

Proof. We fix $t \geq 0$. By Lemmas 2.4 and 4.3, it suffices to prove that, for almost every $x$, $f^{N}(t, x)$ converges to $f^{\infty}(t, x)$ defined by

$$
f^{\infty}(t, x):=\int_{0}^{t} \exp \left(\mathrm{i} \theta \sum_{l=1}^{m} x_{l}\right) g_{0}(\tilde{h}(\theta))^{m}\left|x_{1} \cdots x_{m}\right|^{1 / 2-\tilde{h}(\theta)} \mathrm{d} \theta .
$$

We let

$$
G_{j, 0}^{N}(x)=g_{0}\left(h_{j}^{N}\right)^{m}\left|x_{1} \cdots x_{m}\right|^{1 / 2-h_{j}^{N}} \quad \text { and } \quad G_{j, 1}^{N}(x)=G_{j}^{N}(x)-G_{j, 0}^{N}(x),
$$

where $G_{j}^{N}(x)$ is defined as in the proof of Lemma 4.3. We also consider the same decomposition $f^{N}=f^{N, 1}-f^{N, 2}$ as in the proof of Lemma 4.3 and we let

$$
f^{N, 1}=f^{N, 1,0}-f^{N, 1,1} \quad \text { and } \quad f^{N, 2}=f^{N, 2,0}-f^{N, 2,1},
$$


where, for $\kappa \in\{0,1\}$,

$$
f^{N, 1, \kappa}(t, x)=1_{(-N a, N a)}(x) \frac{\mathrm{i} \sum_{l=1}^{m} x_{l} / N}{1-\exp \left(-\mathrm{i} \sum_{l=1}^{m} x_{l} / N\right)} G_{\lfloor N t\rfloor, \kappa}^{N}(x) \frac{\exp \left(\mathrm{i}\lfloor N t\rfloor \sum_{l=1}^{m} x_{l} / N\right)-1}{\mathrm{i} \sum_{l=1}^{m} x_{l}}
$$

and

$$
\begin{aligned}
f^{N, 2, \kappa}(t, x)= & 1_{(-N a, N a)}(x) \frac{\mathrm{i} \sum_{l=1}^{m} x_{l} / N}{1-\exp \left(-\mathrm{i} \sum_{l=1}^{m} x_{l} / N\right)} \\
& \times \sum_{j=1}^{\lfloor N t\rfloor} \frac{\exp \left(\mathrm{i}(j-1) \sum_{l=1}^{m} x_{l} / N\right)-1}{\mathrm{i} \sum_{l=1}^{m} x_{l}}\left(G_{j, \kappa}^{N}(x)-G_{j-1, \kappa}^{N}(x)\right) .
\end{aligned}
$$

Because $h$ and $g_{0}$ are continuously differentiable, we get, for almost every $x$,

$$
\lim _{N \rightarrow \infty} f^{N, 1,0}(t, x)=g_{0}(\tilde{h}(t))^{m} \frac{\exp \left(\mathrm{i} t \sum_{l=1}^{m} x_{l}\right)-1}{\mathrm{i}\left|x_{1} \cdots x_{m}\right|^{\tilde{h}(t)-1 / 2} \sum_{l=1}^{m} x_{l}}
$$

and

$$
\lim _{N \rightarrow \infty} f^{N, 2,0}(t, x)=\left.\int_{0}^{t} \frac{\exp \left(\mathrm{i} \theta \sum_{l=1}^{m} x_{l}\right)-1}{\mathrm{i} \sum_{l=1}^{m} x_{l}} \tilde{h}^{\prime}(\theta) \frac{\partial}{\partial H}\left(\frac{g_{0}(H)^{m}}{\left|x_{1} \cdots x_{m}\right|^{H-1 / 2}}\right)\right|_{H=\tilde{h}(\theta)} \mathrm{d} \theta,
$$

so that

$$
\lim _{N \rightarrow \infty}\left(f^{N, 1,0}(t, x)-f^{N, 2,0}(t, x)\right)=f^{\infty}(t, x) .
$$

We now deal with $f^{N, 1,1}$ and $f^{N, 2,1}$. We write $G_{j, 1}^{N}(x)$ as

$$
G_{j, 1}^{N}(x)=\left|x_{1} \cdots x_{m}\right|^{1 / 2-h_{j}^{N}} \sum_{k=1}^{m} g_{1}\left(h_{j}^{N}, \frac{x_{k}}{N}\right) g_{0}\left(h_{j}^{N}\right)^{k-1} \prod_{l=k+1}^{m} g\left(h_{j}^{N}, \frac{x_{l}}{N}\right) .
$$

Then, by Lemma 4.1 and because $g_{0}$ and $g$ are bounded, there exist a constant $M_{3}>0$ and a function $\tilde{f}_{t, 3} \in L^{2}\left(\mathbb{R}^{m}, \mathbb{R}\right)$ such that, for almost every $x$,

$$
\left|f^{N, 1,1}(t, x)\right| \leq M_{3} \tilde{f}_{t, 3}(x) \sum_{k=1}^{m} \sup _{H \in[\min \tilde{h}, \max \tilde{h}]}\left|g_{1}\left(H, \frac{x_{k}}{N}\right)\right|,
$$

so that $\lim _{N \rightarrow \infty} f^{N, 1,1}(t, x)=0$. Similarly, by Lemma 4.1, there exist a constant $M_{4}>0$ and a function $\tilde{f}_{t, 4} \in L^{2}\left(\mathbb{R}^{m}, \mathbb{R}\right)$ such that, for almost every $x$,

$$
\left|f^{N, 2,1}(t, x)\right| \leq M_{4} \tilde{f}_{t, 4}(x) \sum_{k=1}^{m} \sup _{H \in[\min \tilde{h}, \max \tilde{h}]}\left(\left|g_{1}\left(H, \frac{x_{k}}{N}\right)\right|+\left|\frac{\partial g_{1}}{\partial H}\left(H, \frac{x_{k}}{N}\right)\right|\right) .
$$

Therefore, $\lim _{N \rightarrow \infty} f^{N, 2,1}(t, x)=0$ and

$$
\lim _{N \rightarrow \infty}\left(f^{N, 1,1}(t, x)-f^{N, 2,1}(t, x)\right)=0,
$$

which concludes the proof. 
The following lemma states that the convergence of $\left\{S_{\phi, h}^{N}\right\}_{N \in \mathbb{N}}$ can be reduced to the convergence of $\left\{S_{P_{m}, h}^{N}\right\}_{N \in \mathbb{N}}$ and, as a consequence of Lemma 4.2, to the convergence of $\left\{\tilde{S}_{m, h}^{N}\right\}_{N \in \mathbb{N}}$.

Lemma 4.5. For every $t \geq 0$,

$$
\lim _{N \rightarrow \infty} \mathbb{E}\left[\left(S_{\phi, h}^{N}(t)-\frac{\left\langle\phi, P_{m}\right\rangle}{m !} S_{P_{m}, h}^{N}(t)\right)^{2}\right]=0 .
$$

Proof. By (2.1) we have

$$
\begin{aligned}
& \mathbb{E}\left[\left(S_{\phi, h}^{N}(t)-\frac{\left\langle\phi, P_{m}\right\rangle}{m !} S_{P_{m}, h}^{N}(t)\right)^{2}\right] \\
& \quad=\mathbb{E}\left[\left(\sum_{j=1}^{\lfloor N t\rfloor} \frac{1}{N^{h(j / N)}} \sum_{n=m+1}^{\infty} \frac{\left\langle\phi, P_{n}\right\rangle}{n !} P_{n}\left(X_{j}\left(h_{j}^{N}\right)\right)\right)^{2}\right] \\
& =\sum_{j=1}^{\lfloor N t\rfloor} \sum_{k=1}^{\lfloor N t\rfloor} \frac{1}{N^{h(j / N)+h(k / N)}} \sum_{n=m+1}^{\infty} \frac{\left\langle\phi, P_{n}\right\rangle^{2}}{(n !)^{2}} \mathbb{E}\left[P_{n}\left(X_{j}\left(h_{j}^{N}\right)\right) P_{n}\left(X_{k}\left(h_{k}^{N}\right)\right)\right] .
\end{aligned}
$$

By (2.2) and (3.2), we obtain, for every $n \geq m+1$,

$$
\mathbb{E}\left[P_{n}\left(X_{j}\left(h_{j}^{N}\right)\right) P_{n}\left(X_{k}\left(h_{k}^{N}\right)\right)\right]=n ! \mathbb{E}\left[X_{j}\left(h_{j}^{N}\right) X_{k}\left(h_{k}^{N}\right)\right]^{n} \leq n !\left|\mathbb{E}\left[X_{j}\left(h_{j}^{N}\right) X_{k}\left(h_{k}^{N}\right)\right]\right|^{m+1},
$$

so that

$$
\begin{aligned}
& \mathbb{E}\left[\left(S_{\phi, h}^{N}(t)-\frac{\left\langle\phi, P_{m}\right\rangle}{m !} S_{P_{m}, h}^{N}(t)\right)^{2}\right] \\
& \quad \leq\left(\sum_{n=m+1}^{\infty} \frac{\left\langle\phi, P_{n}\right\rangle^{2}}{n !}\right) \sum_{j=1}^{\lfloor N t\rfloor} \sum_{k=1}^{\lfloor N t\rfloor} \frac{\left|\mathbb{E}\left[X_{j}\left(h_{j}^{N}\right) X_{k}\left(h_{k}^{N}\right)\right]\right|^{m+1}}{N^{h(j / N)+h(k / N)}} .
\end{aligned}
$$

Let $\eta>0$. By (3.1) and (3.3), there exists $N_{\eta} \in \mathbb{N}^{*}$ such that, for $|j-k|>N_{\eta}$ and $N \in \mathbb{N}^{*}$, $\left|\mathbb{E}\left[X_{j}\left(h_{j}^{N}\right) X_{k}\left(h_{k}^{N}\right)\right]\right| \leq \eta$. Therefore,

$$
\begin{aligned}
& \sum_{j=1}^{\lfloor N t\rfloor} \sum_{k=1}^{\lfloor N t\rfloor} \frac{\left|\mathbb{E}\left[X_{j}\left(h_{j}^{N}\right) X_{k}\left(h_{k}^{N}\right)\right]\right|^{m+1}}{N^{h(j / N)+h(k / N)}} \\
& \quad \leq \sum_{j=1}^{\lfloor N t\rfloor} \sum_{k=1}^{\lfloor N t\rfloor} \frac{1_{\left\{|j-k| \leq N_{\eta}\right\}}}{N^{h(j / N)+h(k / N)}}+\eta \sum_{j=1}^{\lfloor N t\rfloor} \sum_{k=1}^{\lfloor N t\rfloor} 1_{\{|j-k| \geq 1\}} \frac{\left|\mathbb{E}\left[X_{j}\left(h_{j}^{N}\right) X_{k}\left(h_{k}^{N}\right)\right]\right|^{m}}{N^{h(j / N)+h(k / N)}} .
\end{aligned}
$$

There exists $C_{1}(\eta)>0$ such that

$$
\sum_{j=1}^{\lfloor N t\rfloor} \sum_{k=1}^{\lfloor N t\rfloor} \frac{1_{\left\{|j-k| \leq N_{\eta}\right\}}}{N^{h(j / N)+h(k / N)}} \leq \frac{C_{1}(\eta)}{N^{2 \min h-1}} .
$$

Moreover, by (3.1) and (3.3), there exists a constant $C_{2}>0$, which is independent on $\eta$, such that, for all $j, k$, and $N$,

$$
\left|\mathbb{E}\left[X_{j}\left(h_{j}^{N}\right) X_{k}\left(h_{k}^{N}\right)\right]\right| \leq C_{2}|j-k|^{h_{j}^{N}+h_{k}^{N}-2} .
$$


We then obtain

$$
\begin{aligned}
& \sum_{j=1}^{\lfloor N t\rfloor} \sum_{k=1}^{\lfloor N t\rfloor} \frac{\left|\mathbb{E}\left[X_{j}\left(h_{j}^{N}\right) X_{k}\left(h_{k}^{N}\right)\right]\right|^{m+1}}{N^{h(j / N)+h(k / N)}} \\
& \quad \leq \frac{C_{1}(\eta)}{N^{2 \min h-1}}+\frac{\eta C_{2}}{N^{2}} \sum_{j=1}^{\lfloor N t\rfloor} \sum_{k=1}^{\lfloor N t\rfloor} 1_{\{|j-k| \geq 1\}}\left|\frac{j-k}{N}\right|^{m\left(h_{j}^{N}+h_{k}^{N}-2\right)}
\end{aligned}
$$

Hence, for every $\eta>0$,

$$
\begin{aligned}
\limsup _{N \rightarrow \infty} \mathbb{E}\left[\left(S_{\phi, h}^{N}(t)-\frac{\left\langle\phi, P_{m}\right\rangle}{m !} S_{P_{m}, h}^{N}(t)\right)^{2}\right] \\
\quad \leq \eta C_{2}\left(\sum_{n=m+1}^{\infty} \frac{\left\langle\phi, P_{n}\right\rangle^{2}}{n !}\right) \int_{0}^{t} \int_{0}^{t}|\theta-\sigma|^{h(\theta)+h(\sigma)-2} \mathrm{~d} \theta \mathrm{d} \sigma .
\end{aligned}
$$

The constants $\sum_{n=m+1}^{\infty}\left\langle\phi, P_{n}\right\rangle^{2} / n$ ! and $\int_{0}^{t} \int_{0}^{t}|\theta-\sigma|^{h(\theta)+h(\sigma)-2} \mathrm{~d} \theta \mathrm{d} \sigma$ are finite by (2.3) and because $\min h>\frac{1}{2}$, respectively. This concludes the proof.

We conclude this subsection with the following lemma.

Lemma 4.6. As $N \rightarrow \infty$, the finite-dimensional distributions of $S_{\phi, h}^{N}$ converge to those of $S_{m, h}$, which can be defined by

$$
S_{m, h}(t):=\frac{\left\langle\phi, P_{m}\right\rangle}{m !} \int_{\mathbb{R}^{m}} f^{\infty}\left(t, x_{1}, \ldots, x_{m}\right) \mathrm{d} \hat{B}_{x_{1}} \cdots \mathrm{d} \hat{B}_{x_{m}}
$$

for every $t \geq 0$.

Proof. We fix $n \in \mathbb{N},\left(t_{1}, \ldots, t_{n}\right) \in[0, \infty)^{n}$, and a Lipschitz bounded function $\Psi: \mathbb{R}^{n} \rightarrow \mathbb{R}$. We define $\phi_{m}=\left\langle\phi, P_{m}\right\rangle / m$ !. We have

$$
\left|\mathbb{E}\left[\Psi\left(S_{\phi, h}^{N}\left(t_{1}\right), \ldots, S_{\phi, h}^{N}\left(t_{n}\right)\right)\right]-\mathbb{E}\left[\Psi\left(S_{m, h}\left(t_{1}\right), \ldots, S_{m, h}\left(t_{n}\right)\right)\right]\right| \leq E_{1}^{N}+E_{2}^{N},
$$

where

$$
E_{1}^{N}=\left|\mathbb{E}\left[\Psi\left(S_{\phi, h}^{N}\left(t_{1}\right), \ldots, S_{\phi, h}^{N}\left(t_{n}\right)\right)-\Psi\left(\phi_{m} S_{P_{m}, h}^{N}\left(t_{1}\right), \ldots, \phi_{m} S_{P_{m}, h}^{N}\left(t_{n}\right)\right)\right]\right|
$$

and

$$
E_{2}^{N}=\left|\mathbb{E}\left[\Psi\left(\phi_{m} S_{P_{m}, h}^{N}\left(t_{1}\right), \ldots, \phi_{m} S_{P_{m}, h}^{N}\left(t_{n}\right)\right)\right]-\mathbb{E}\left[\Psi\left(S_{m, h}\left(t_{1}\right), \ldots, S_{m, h}\left(t_{n}\right)\right)\right]\right| .
$$

Because $\Psi$ is Lipschitz and by the Cauchy-Schwarz inequality, there exists $C_{1}>0$ such that, for every $N$,

$$
E_{1}^{N} \leq C_{1} \sum_{j=1}^{n} \sqrt{\mathbb{E}\left[\left(S_{\phi, h}^{N}\left(t_{j}\right)-\phi_{m} S_{P_{m}, h}^{N}\left(t_{j}\right)\right)^{2}\right]} .
$$

Then, by Lemma 4.5,

$$
\lim _{N \rightarrow \infty} E_{1}^{N}=0
$$


By Lemma 4.2 we have

$$
E_{2}^{N}=\left|\mathbb{E}\left[\Psi\left(\phi_{m} \tilde{S}_{m, h}^{N}\left(t_{1}\right), \ldots, \phi_{m} \tilde{S}_{m, h}^{N}\left(t_{n}\right)\right)-\Psi\left(S_{m, h}\left(t_{1}\right), \ldots, S_{m, h}\left(t_{n}\right)\right)\right]\right| .
$$

Thus, as in the $E_{1}^{N}$ case, there exists $C_{2}>0$ such that, for every $N$,

$$
E_{2}^{N} \leq C_{2} \sum_{j=1}^{n} \sqrt{\mathbb{E}\left[\left(\phi_{m} \tilde{S}_{m, h}^{N}\left(t_{j}\right)-S_{m, h}\left(t_{j}\right)\right)^{2}\right]} .
$$

As a consequence, by Lemma 4.4 ,

$$
\lim _{N \rightarrow \infty} E_{2}^{N}=0
$$

Combining (4.5), (4.6), and (4.7) completes the proof.

\subsection{Continuity, local self-similarity, and local Hölder exponent of the limit}

In this subsection we first prove the convergence of the finite-dimensional distributions of $\left\{T_{m, h, t}^{\varepsilon}\right\}_{\varepsilon>0}$ for every $t$. Then, we prove the continuity of $S_{m, h}$ and the tightness of $\left\{T_{m, h, t}^{\varepsilon}\right\}_{\varepsilon>0}$ in $\mathcal{C}([0, \infty))$ to deduce the local self-similarity property. Finally, we deal with the local Hölder exponent of $S_{m, h}$.

Making the substitution $\theta \rightarrow \varepsilon \theta+t$, we obtain, for every $u \geq 0$,

$$
T_{m, h, t}^{\varepsilon}(u)=\int_{\mathbb{R}^{m}} \exp \left(\mathrm{i} t \sum_{l=1}^{m} x_{l}\right) \psi_{1}(t, u, x, \varepsilon) \mathrm{d} \hat{B}_{x_{1}} \cdots \mathrm{d} \hat{B}_{x_{m}},
$$

where

$$
\psi_{1}(t, u, x, \varepsilon)=\varepsilon^{1-h(t)} \int_{0}^{u} \exp \left(\mathrm{i} \varepsilon \theta \sum_{l=1}^{m} x_{l}\right) \tilde{g}(\varepsilon \theta+t)\left|x_{1} \cdots x_{m}\right|^{1 / 2-\tilde{h}(\varepsilon \theta+t)} \mathrm{d} \theta .
$$

By (2.6) and (2.7), we have

$$
\left\{T_{m, h, t}^{\varepsilon}(u)\right\}_{u \geq 0} \stackrel{\mathrm{D}}{=}\left\{\int_{\mathbb{R}^{m}} \psi_{2}(t, u, x, \varepsilon) \mathrm{d} \hat{B}_{x_{1}} \cdots \mathrm{d} \hat{B}_{x_{m}}\right\}_{u \geq 0}
$$

with

$$
\psi_{2}(t, u, x, \varepsilon)=\int_{0}^{u} \varepsilon^{m(\tilde{h}(\varepsilon \theta+t)-\tilde{h}(t))} \exp \left(\mathrm{i} \theta \sum_{l=1}^{m} x_{l}\right) \tilde{g}(\varepsilon \theta+t)\left|x_{1} \cdots x_{m}\right|^{1 / 2-\tilde{h}(\varepsilon \theta+t)} \mathrm{d} \theta .
$$

For all $u$ and $t$ and almost every $x$,

$$
\lim _{\varepsilon \rightarrow 0} \psi_{2}(t, u, x, \varepsilon)=\tilde{g}(t) \frac{\exp \left(\mathrm{i} u \sum_{l=1}^{m} x_{l}\right)-1}{\mathrm{i}\left(\sum_{l=1}^{m} x_{l}\right)\left|x_{1} \cdots x_{m}\right|^{\tilde{h}(t)-1 / 2}} .
$$

By an integration by parts,

$$
\begin{aligned}
\psi_{2}(t, u, x, \varepsilon)= & \varepsilon^{m(\tilde{h}(\varepsilon u+t)-\tilde{h}(t))} \tilde{g}(\varepsilon u+t) \frac{\exp \left(\mathrm{i} u \sum_{l=1}^{m} x_{l}\right)-1}{\mathrm{i}\left(\sum_{l=1}^{m} x_{l}\right)\left|x_{1} \cdots x_{m}\right|^{\tilde{h}(\varepsilon u+t)-1 / 2}} \\
& +\varepsilon \int_{0}^{u} \varepsilon^{m(\tilde{h}(\varepsilon \theta+t)-\tilde{h}(t))} \frac{\exp \left(\mathrm{i} \theta \sum_{l=1}^{m} x_{l}\right)-1}{\mathrm{i}\left(\sum_{l=1}^{m} x_{l}\right)\left|x_{1} \cdots x_{m}\right|^{\tilde{h}(\varepsilon \theta+t)-1 / 2}} \\
& \times\left\{\tilde{g}^{\prime}(\varepsilon \theta+t)+\tilde{h}^{\prime}(\varepsilon \theta+t) \tilde{g}(\varepsilon \theta+t)\left(\ln \left(\varepsilon^{m}\right)-\ln \left|x_{1} \cdots x_{m}\right|\right)\right\} \mathrm{d} \theta .
\end{aligned}
$$


As a consequence of the identity above and because of Lemma 4.1, there exists a function $\psi_{3, t, u} \in L^{2}\left(\mathbb{R}^{m}, \mathbb{R}_{+}\right)$such that, for every $x \in \mathbb{R}^{m}$,

$$
\left|\psi_{2}(t, u, x, \varepsilon)\right| \leq \psi_{3, t, u}(x) .
$$

Because $\psi_{3, t, u}$ is square integrable and by Lemma 2.4, this proves the convergence of the finite-dimensional distributions of $T_{m, h, t}^{\varepsilon}$.

To prove the continuity of $S_{m, h}$, we use the Kolmogorov lemma. Let $T>0$. As previously, by Lemma 4.1 again, there exists $C>0$ such that, for all $0 \leq s<t \leq T$,

$$
\begin{aligned}
\mathbb{E}\left[\left(S_{m, h}(t)-S_{m, h}(s)\right)^{2}\right] & =m !(t-s)^{2 h(s)} \int_{\mathbb{R}^{m}}\left(\psi_{2}(s, 1, x, t-s)\right)^{2} \mathrm{~d} x_{1} \cdots \mathrm{d} x_{m} \\
& \leq C(t-s)^{2 h(s)},
\end{aligned}
$$

which concludes the proof of the continuity of $S_{m, h}$.

Moreover, in a similar way, we prove that, for every compact set $U \subset[0, \infty)$, there exists a constant $C_{U}>0$ such that, for all $u$ and $v$ in $U$ such that $|u-v|<1$, we have

$$
\mathbb{E}\left[\left(\frac{S_{m, h}(t+\varepsilon u)-S_{m, h}(t+\varepsilon v)}{\varepsilon^{h(t)}}\right)^{2}\right] \leq C_{U}|u-v|^{2 h(t)} .
$$

This proves the tightness of the family $\left\{T_{m, h, t}^{\varepsilon}\right\}_{\varepsilon>0}$ in $\mathcal{C}([0, \infty))$ by the Kolmogorov lemma [3], and then the local self-similarity property of $S_{m, h}$.

Finally, for each $t_{0} \geq 0$, we deal with the local Hölder exponent of $S_{m, h}$ around $t_{0}$, which is denoted by $\alpha_{S_{m, h}}\left(t_{0}\right)$. By (4.8) and Lemma 2.5, for every $p \in \mathbb{N}^{*}$, there exists $C_{p}>0$ such that, for all $s \leq t$ in a neighborhood of $t_{0}$,

$$
\mathbb{E}\left[\left(S_{m, h}(t)-S_{m, h}(s)\right)^{2 p}\right] \leq C_{p}(t-s)^{2 p \inf _{[s, t]} h} .
$$

By the Kolmogorov lemma, this implies that $\alpha_{S_{m, h}}\left(t_{0}\right) \geq h\left(t_{0}\right)$. Using the local self-similarity of $S_{m, h}$ and proceeding as in [1], we prove that $\alpha_{S_{m, h}}\left(t_{0}\right) \leq h\left(t_{0}\right)$, which gives $\alpha_{S_{m, h}}\left(t_{0}\right)=h\left(t_{0}\right)$ and concludes this subsection.

\subsection{Tightness}

Because of Theorem 15.6 of [3], it is enough to show that there exist $C>0$ and $\gamma>1$ such that, for all $t_{1}, t_{2}, t_{3} \in[0, T]$ satisfying $t_{1}<t_{2}<t_{3}$ and $t_{3}-t_{1}<1$, we have

$$
\mathbb{E}\left[\left|S_{\phi, h}^{N}\left(t_{3}\right)-S_{\phi, h}^{N}\left(t_{2}\right)\right|\left|S_{\phi, h}^{N}\left(t_{2}\right)-S_{\phi, h}^{N}\left(t_{1}\right)\right|\right] \leq C\left(t_{3}-t_{1}\right)^{\gamma} .
$$

First, we assume that $t_{3}-t_{1}<1 / N$. Hence, $\left\lfloor N t_{1}\right\rfloor=\left\lfloor N t_{2}\right\rfloor$ or $\left\lfloor N t_{2}\right\rfloor=\left\lfloor N t_{3}\right\rfloor$, which gives

$$
\mathbb{E}\left[\left|S_{\phi, h}^{N}\left(t_{3}\right)-S_{\phi, h}^{N}\left(t_{2}\right)\right|\left|S_{\phi, h}^{N}\left(t_{2}\right)-S_{\phi, h}^{N}\left(t_{1}\right)\right|\right]=0 .
$$

Then, we assume that $1 / N \leq t_{3}-t_{1}<1$. By the Cauchy-Schwarz inequality, we have

$$
\begin{aligned}
& \mathbb{E}\left[\left|S_{\phi, h}^{N}\left(t_{3}\right)-S_{\phi, h}^{N}\left(t_{2}\right)\right|\left|S_{\phi, h}^{N}\left(t_{2}\right)-S_{\phi, h}^{N}\left(t_{1}\right)\right|\right] \\
& \quad \leq \sqrt{\mathbb{E}\left[\left|S_{\phi, h}^{N}\left(t_{3}\right)-S_{\phi, h}^{N}\left(t_{2}\right)\right|^{2}\right]} \sqrt{\mathbb{E}\left[\left|S_{\phi, h}^{N}\left(t_{2}\right)-S_{\phi, h}^{N}\left(t_{1}\right)\right|^{2}\right]} .
\end{aligned}
$$

Consequently, it is enough to prove that there exist $C>0$ and $\gamma>1$ such that, for $(s, t)=$ $\left(t_{1}, t_{2}\right)$ or $\left(t_{2}, t_{3}\right)$,

$$
\mathbb{E}\left[\left|S_{\phi, h}^{N}(t)-S_{\phi, h}^{N}(s)\right|^{2}\right] \leq C\left(t_{3}-t_{1}\right)^{\gamma}
$$


Proceeding as in the proof of Lemma 4.5, we obtain

$$
\begin{aligned}
& \mathbb{E}\left[\left|S_{\phi, h}^{N}(t)-S_{\phi, h}^{N}(s)\right|^{2}\right] \\
& \leq\left(\sum_{n=m}^{\infty} \frac{\left\langle\phi, P_{n}\right\rangle^{2}}{n !}\right) \sum_{j=\lfloor N s\rfloor+1}^{\lfloor N t\rfloor} \sum_{k=\lfloor N s\rfloor+1}^{\lfloor N t\rfloor} \frac{\mathbb{E}\left[X_{j}\left(h_{j}^{N}\right) X_{k}\left(h_{k}^{N}\right)\right]^{m}}{N^{h(j / N)+h(k / N)}} .
\end{aligned}
$$

Because of (4.4), there exists $C_{1}>0$ such that

$$
\begin{aligned}
& \sum_{j=\lfloor N s\rfloor+1}^{\lfloor N t\rfloor} \sum_{k=\lfloor N s\rfloor+1}^{\lfloor N t\rfloor} \frac{\mathbb{E}\left[X_{j}\left(h_{j}^{N}\right) X_{k}\left(h_{k}^{N}\right)\right]^{m}}{N^{h(j / N)+h(k / N)}} \\
& \quad \leq \sum_{j=\left\lfloor N t_{1}\right\rfloor+1}^{\left\lfloor N t_{3}\right\rfloor} \frac{\left|\mathbb{E}\left[X_{0}\left(h_{j}^{N}\right)^{2}\right]\right|^{m}}{N^{h(j / N)+h(k / N)}}+C_{1} \sum_{j, k=\left\lfloor N t_{1}\right\rfloor+1, j \neq k}^{\left\lfloor N t_{3}\right\rfloor} \frac{|j-k|^{m\left(h_{j}^{N}+h_{k}^{N}-2\right)}}{N^{h(j / N)+h(k / N)}} \\
& \quad \leq C_{2} \frac{\left\lfloor N t_{3}\right\rfloor-\left\lfloor N t_{1}\right\rfloor}{N^{2 \min h}}+\frac{C_{1}}{N^{2}} \sum_{j, k=\left\lfloor N t_{1}\right\rfloor+1, j \neq k}^{\left\lfloor N t_{3}\right\rfloor}\left|\frac{j-k}{N}\right|^{2 \min h-2},
\end{aligned}
$$

where $C_{2}=\max _{H \in[\min h, \max h]}\left|\mathbb{E}\left[X_{0}(H)^{2}\right]\right|^{m}$. We have

$$
\frac{\left\lfloor N t_{3}\right\rfloor-\left\lfloor N t_{1}\right\rfloor}{N^{2 \min h}} \leq \frac{t_{3}-t_{1}}{N^{2 \min h-1}}+\frac{1}{N^{2 \min h}} \leq 2\left(t_{3}-t_{1}\right)^{2 \min h}
$$

because $1 / N \leq t_{3}-t_{1}$. Moreover,

$$
\begin{aligned}
\frac{1}{N^{2}} \sum_{j, k=\left\lfloor N t_{1}\right\rfloor+1, j \neq k}^{\left\lfloor N t_{3}\right\rfloor}\left|\frac{j-k}{N}\right|^{2 \min h-2} & \leq C_{3} \int_{s}^{t} \mathrm{~d} \theta \int_{t_{1}}^{t_{3}} \mathrm{~d} \sigma|\theta-\sigma|^{2 \min h-2} \\
& \leq \frac{C_{3}\left(t_{3}-t_{1}\right)^{2 \min h}}{(2 \min h-1) \min h},
\end{aligned}
$$

where $C_{3}>0$ does not depend on $\left(N, t_{1}, t_{3}\right)$. Combining (4.16), (4.15), (4.14), and (4.13), we get (4.12). Because of (4.11) and (4.10), this concludes the proof of (4.9).

\section{Appendix A. Technical lemma}

This section is devoted to the proof of a lemma that deals with the asymptotic behavior of a covariance function.

Lemma A.1. Let $X=\left\{X_{n}(H)\right\}_{(n, H) \in \mathbb{N} \times(b, 1)}$ be a random field as defined by (3.1). For every compact set $K \subset(b, 1)$,

$$
\lim _{n \rightarrow \infty} \sup _{\left(H_{1}, H_{2}\right) \in K^{2}}\left|n^{2-H_{1}-H_{2}} \mathbb{E}\left[X_{n}\left(H_{1}\right) X_{0}\left(H_{2}\right)\right]-R\left(H_{1}, H_{2}\right)\right|=0,
$$

where, for every $\left(H_{1}, H_{2}\right) \in(b, 1)^{2}$,

$$
R\left(H_{1}, H_{2}\right)=g_{0}\left(H_{1}\right) g_{0}\left(H_{2}\right) \int_{\mathbb{R}} \exp (\mathrm{i} x)|x|^{1-H_{1}-H_{2}} \mathrm{~d} x .
$$


Proof. Let $K$ be a compact set in $(b, 1)$. We fix $\left(H_{1}, H_{2}\right) \in K^{2}$ and $n \in \mathbb{N}^{*}$. We have

$$
\begin{aligned}
\mathbb{E}\left[X_{n}\left(H_{1}\right) X_{0}\left(H_{2}\right)\right] & =\int_{-a}^{a} \exp (\mathrm{i} n x) g\left(H_{1}, x\right) \overline{g\left(H_{2}, x\right)}|x|^{1-H_{1}-H_{2}} \mathrm{~d} x \\
& =n^{H_{1}+H_{2}-2} \int_{-n a}^{n a} \exp (\mathrm{i} n x) g\left(H_{1}, \frac{x}{n}\right) \overline{g\left(H_{2}, \frac{x}{n}\right)}|x|^{1-H_{1}-H_{2}} \mathrm{~d} x .
\end{aligned}
$$

Setting

$$
\psi_{H_{1}, H_{2}}(y)=g\left(H_{1}, y\right) \overline{g\left(H_{2}, y\right)}-g_{0}\left(H_{1}\right) g_{0}\left(H_{2}\right)
$$

for every $y \in \mathbb{R}$, we prove hereafter that

$$
\left.\lim _{n \rightarrow \infty} \sup _{\left(H_{1}, H_{2}\right) \in K^{2}}\left|g_{0}\left(H_{1}\right) g_{0}\left(H_{2}\right) \int_{-n a}^{n a} \exp (\mathrm{i} x)\right| x\right|^{1-H_{1}-H_{2}} \mathrm{~d} x-R\left(H_{1}, H_{2}\right) \mid=0
$$

and

$$
\left.\lim _{n \rightarrow \infty} \sup _{\left(H_{1}, H_{2}\right) \in K^{2}}\left|\int_{-n a}^{n a} \exp (\mathrm{i} x) \psi_{H_{1}, H_{2}}\left(\frac{x}{n}\right)\right| x\right|^{1-H_{1}-H_{2}} \mathrm{~d} x \mid=0 .
$$

By an integration by parts, we have

$$
\begin{aligned}
& \frac{1}{2}\left(\int_{-n a}^{n a} \exp (\mathrm{i} x)|x|^{1-H_{1}-H_{2}} \mathrm{~d} x-\int_{-\infty}^{\infty} \exp (\mathrm{i} x)|x|^{1-H_{1}-H_{2}} \mathrm{~d} x\right) \\
& \quad=-\int_{n a}^{\infty} \cos (x) x^{1-H_{1}-H_{2}} \mathrm{~d} x \\
& \quad=\sin (n a)(n a)^{1-H_{1}-H_{2}}+\left(1-H_{1}-H_{2}\right) \int_{n a}^{\infty} \sin (x) x^{-H_{1}-H_{2}} \mathrm{~d} x
\end{aligned}
$$

Hence, we get

$$
\begin{aligned}
& \left.\sup _{\left(H_{1}, H_{2}\right) \in K^{2}}\left|g_{0}\left(H_{1}\right) g_{0}\left(H_{2}\right) \int_{-n a}^{n a} \exp (\mathrm{i} x)\right| x\right|^{1-H_{1}-H_{2}} \mathrm{~d} x-R\left(H_{1}, H_{2}\right) \mid \\
& \quad \leq 2 \sup _{\left(H_{1}, H_{2}\right) \in K^{2}}\left|g_{0}\left(H_{1}\right) g_{0}\left(H_{2}\right)\left(|n a|^{1-H_{1}-H_{2}}+\int_{n a}^{\infty}|x|^{-H_{1}-H_{2}} \mathrm{~d} x\right)\right|,
\end{aligned}
$$

which implies (A.1) because $K \subset\left(\frac{1}{2}, 1\right)$.

Now we prove (A.2). Again by integration by parts, we obtain

$$
\begin{aligned}
& \int_{0}^{n a} \exp (\mathrm{i} x) \psi_{H_{1}, H_{2}}\left(\frac{x}{n}\right) x^{1-H_{1}-H_{2}} \mathrm{~d} x \\
& =\mathrm{i}\left(1-\mathrm{e}^{\mathrm{i} n a}\right) \psi_{H_{1}, H_{2}}(a)(n a)^{1-H_{1}-H_{2}}-\frac{\mathrm{i}}{n} \int_{0}^{n a}\left(1-\mathrm{e}^{\mathrm{i} x}\right) \psi_{H_{1}, H_{2}}^{\prime}\left(\frac{x}{n}\right) x^{1-H_{1}-H_{2}} \mathrm{~d} x \\
& \quad+\mathrm{i}\left(H_{1}+H_{2}-1\right) \int_{0}^{n a}\left(1-\mathrm{e}^{\mathrm{i} x}\right) \psi_{H_{1}, H_{2}}\left(\frac{x}{n}\right) x^{-H_{1}-H_{2}} \mathrm{~d} x .
\end{aligned}
$$

Obviously, we have

$$
\begin{aligned}
& \sup _{\left(H_{1}, H_{2}\right) \in K^{2}}\left|\mathrm{i}\left(1-\mathrm{e}^{\mathrm{i} n a}\right) \psi_{H_{1}, H_{2}}(a)(n a)^{1-H_{1}-H_{2}}\right| \\
& \quad \leq 2 \sup _{\left(H_{1}, H_{2}\right) \in K^{2}}\left|\psi_{H_{1}, H_{2}}(a)\right| \sup _{\left(H_{1}, H_{2}\right) \in K^{2}}\left|(n a)^{1-H_{1}-H_{2}}\right| \\
& \quad \rightarrow 0 \text { as } n \rightarrow \infty .
\end{aligned}
$$


By a change of variable we get

$$
\begin{aligned}
& \sup _{\left(H_{1}, H_{2}\right) \in K^{2}}\left|\frac{\mathrm{i}}{n} \int_{0}^{n a}\left(1-\mathrm{e}^{\mathrm{i} x}\right) \psi_{H_{1}, H_{2}}^{\prime}\left(\frac{x}{n}\right) x^{1-H_{1}-H_{2}} \mathrm{~d} x\right| \\
& \quad \leq \sup _{\left(H_{1}, H_{2}\right) \in K^{2}}\left|\int_{0}^{a} \psi_{H_{1}, H_{2}}^{\prime}(x) x^{1-H_{1}-H_{2}} \mathrm{~d} x\right| \sup _{\left(H_{1}, H_{2}\right) \in K^{2}}\left|n^{1-H_{1}-H_{2}}\right| \\
& \quad \rightarrow 0 \text { as } n \rightarrow \infty
\end{aligned}
$$

It remains to prove that

$$
\lim _{n \rightarrow \infty} \sup _{\left(H_{1}, H_{2}\right) \in K^{2}}\left|\left(H_{1}+H_{2}-1\right) \int_{0}^{n a}\left(1-\mathrm{e}^{\mathrm{i} x}\right) \psi_{H_{1}, H_{2}}\left(\frac{x}{n}\right) x^{-H_{1}-H_{2}} \mathrm{~d} x\right|=0 .
$$

By the assumptions on $g_{1}$, for almost every $x \in \mathbb{R}$,

$$
\lim _{n \rightarrow \infty} 1_{(0, n a)}(x)\left(1-\mathrm{e}^{\mathrm{i} x}\right) \sup _{\left(H_{1}, H_{2}\right) \in K^{2}}\left|\left(H_{1}+H_{2}-1\right) \psi_{H_{1}, H_{2}}\left(\frac{x}{n}\right) x^{-H_{1}-H_{2}}\right|=0 .
$$

Moreover,

$$
\begin{aligned}
& 1_{(0, n a)}(x)\left(1-\mathrm{e}^{\mathrm{i} x}\right) \sup _{\left(H_{1}, H_{2}\right) \in K^{2}}\left|\left(H_{1}+H_{2}-1\right) \psi_{H_{1}, H_{2}}\left(\frac{x}{n}\right) x^{-H_{1}-H_{2}}\right| \\
& \leq\left(1-\mathrm{e}^{\mathrm{i} x}\right) \sup _{\left(H_{1}, H_{2}\right) \in K^{2}}\left|\left(H_{1}+H_{2}-1\right) x^{-H_{1}-H_{2}}\right| \sup _{\left(H_{1}, H_{2}, y\right) \in K^{2} \times(0, a)}\left|\psi_{H_{1}, H_{2}}(y)\right| .
\end{aligned}
$$

The function $x \mapsto\left(1-\mathrm{e}^{\mathrm{i} x}\right) \sup _{\left(H_{1}, H_{2}\right) \in K^{2}}\left|\left(H_{1}+H_{2}-1\right) x^{-H_{1}-H_{2}}\right|$ is integrable. Then, by the dominated convergence theorem we obtain (A.6). Combining (A.3), (A.4), (A.5), and (A.6), we obtain

$$
\lim _{n \rightarrow \infty} \sup _{\left(H_{1}, H_{2}\right) \in K^{2}}\left|\int_{0}^{n a} \mathrm{e}^{\mathrm{i} x} \psi_{H_{1}, H_{2}}\left(\frac{x}{n}\right) x^{1-H_{1}-H_{2}} \mathrm{~d} x\right|=0 .
$$

Similarly, we obtain

$$
\lim _{n \rightarrow \infty} \sup _{\left(H_{1}, H_{2}\right) \in K^{2}}\left|\int_{-n a}^{0} \mathrm{e}^{\mathrm{i} x} \psi_{H_{1}, H_{2}}\left(\frac{x}{n}\right) x^{1-H_{1}-H_{2}} \mathrm{~d} x\right|=0,
$$

which proves (A.2).

\section{Acknowledgements}

I would like to thank Céline Lacaux for many fruitful discussions about multifractional processes. I also thank the anonymous referee for valuable remarks that made me improve an earlier version of the paper.

\section{References}

[1] Benassi, A., Cohen, S. And Istas, J. (2002). Identification and properties of real harmonizable Lévy motions. Bernoulli 8, 97-115.

[2] Benassi, A., JAFFARD, S. ANd Roux, D. (1997). Elliptic Gaussian random processes. Rev. Math. Iberoamericana 13, 19-90. 
[3] Billingsley, P. (1968). Convergence of Probability Measures. John Wiley, New York.

[4] Cohen, S. And Marty, R. (2008). Invariance principle, multifractional Gaussian processes and long-range dependence. Ann. Inst. H. Poincaré Prob. Statist. 44, 475-489.

[5] Davydov, Y. A. (1970). The invariance principle for stationary processes. Theory Prob. Appl. 15, 487-498.

[6] Dobrushin, R. L. (1979). Gaussian and their subordinated self-similar random generalized fields. Ann. Prob. 7, 1-28.

[7] Dobrushin, R. L. AND MaJor, P. (1979). Non-central limit theorems for nonlinear functionals of Gaussian fields. Z. Wahrscheinlichkeitsth. 50, 27-52.

[8] Itô, K. (1951). Multiple Wiener integral. J. Math. Soc. Japan 3, 157-169.

[9] Lacaux, C. AND Marty, R. (2011). From invariance principles to a class of multifractional fields related to fractional sheets. Preprint. Available at http://hal.archives-ouvertes.fr/hal-00592188.

[10] Marty, R. AND SøLnA, K. (2011). A general framework for waves in random media with long-range correlations. Ann. Appl. Prob. 21, 115-139.

[11] Nelson, E. (1973). The free Markoff field. J. Funct. Anal. 12, 211-227.

[12] Nourdin, I. And Peccati, G. (2012). Normal Approximations Using Malliavin Calculus (Cambridge Tracts Math. 192). Cambridge University Press.

[13] Peltier, R. F. And Lévy VéHel, J. (1995). Multifractional Brownian motion: definition and preliminary results. Preprint. Available at http://hal.inria.fr/inria-00074045/.

[14] Samorodnitsky, G. And Taqqu, M. S. (1994). Stable Non-Gaussian Random Processes. Chapman and Hall, New York.

[15] SLY, A. (2007). Integrated fractional white noise as an alternative to multifractional Brownian motion. J. Appl. Prob. 44, 393-408.

[16] TAQQU, M. S. (1975). Weak convergence to fractional Brownian motion and to the Rosenblatt process. Z. Wahrscheinlichkeitsth. 31, 287-302.

[17] TAQQU, M. S. (1979). Convergence of integrated processes of arbitrary Hermite rank. Z. Wahrscheinlichkeitsth. 50, 53-83. 